\title{
Sleep, an integrated physiological function of living body
}

Hiroyoshi Séi ${ }^{1}$

Published online: 22 August 2018

(c) Japanese Society of Sleep Research 2018

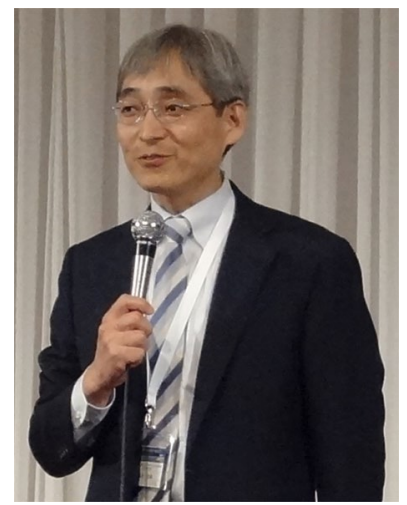

It is my great pleasure to be an associate editor of Sleep and Biological Rhythms from 2018. I feel really honored and strongly responsible for handling valuable manuscripts that will be sent from all over the world. I will continue trying to make this journal achieve more and more progress from now on.

I am teaching physiology at faculty of medicine. Physiology aims to understand the mechanism of living organisms, and we experiment first with the individual animals as whole body beings. I believe that normal sleep can be observed only in the physiologically normal animals; so sleep is thought to be one of the most suitable themes of physiological study.

Currently, one of the sleep-related researches we are conducting in our laboratory is the relationship between sleep homeostasis and lipid metabolism, especially the association with ketone bodies [2, 4]. For hyperlipidemia in which triglyceride levels are high, fibrates such as bezafibrate, which is an artificial ligand of PPAR, are used as therapeutic agents. Bezafibrate mainly acts on PPAR $\alpha$ and activates

Hiroyoshi Séi

sei@tokushima-u.ac.jp

1 Department of Integrative Physiology, Tokushima

University Graduate School of Biomedical Sciences,

Tokushima 770-8503, Japan lipid metabolism. When burning lipids, ketone bodies are produced as final products. When we give bezafibrate to mice, ketone bodies increase in the blood. The mice also show increased delta-wave power during non-REM sleep and increased arousal threshold. In other words, bezafibrate makes non-REM sleep deeper in mice. From these results, we have hypothesized that ketone bodies are involved in the regulation of sleep depth, i.e., homeostasis. After long or exhausting wakefulness, lipid metabolism is activated to produce the additional ATP and then ketone body will increase. This may cause the increase of sleep depth in homeostatic manner.

Another research that we are doing is about the association of mast cells with sleep disorders [1,3]. This is the theme first proposed by Professor Seiji Nishino from Stanford University. Sachiko Chikahisa, a lecturer in our laboratory, studied abroad at his laboratory in 2009-2010 and has collaborated with him until now. Mast cells play a central role in allergic reactions producing and releasing many physiologically active substances such as histamine, serotonin, or prostaglandin. Mast cells also exist in the skull and release physiologically active substances, but their physiological or pathological roles are not well understood. Recently, we found that in sleep disorder model mice, impaired glucose tolerance occurs with the increase in number of intracranial mast cells. Furthermore, it was found that administration of a release inhibitor of mast cells into the cerebral ventricle improves both sleep and glucose tolerance impairment. We are hypothesizing that mast cells present in human brain may also be involved in sleep disorders and various pathologies associated therewith, and we are continuing our research.

In recent years, neuroscience has reached a new era with the rapid progress of its methodology. As for the mechanism of sleep, exciting novel findings are announced one after another. In near future, I expect that a great paper will be published in SBR, with which a breakthrough will occur in sleep science. 


\section{References}

1. Chikahisa S, Harada S, Shimizu N, Shiuchi T, Otsuka A, Nishino $\mathrm{S}$, Séi $\mathrm{H}$. Mast cell involvement in glucose tolerance impairment caused by chronic mild stress with sleep disturbance. Sci Rep. 2017;7(1):13640.

2. Chikahisa S, Shimizu N, Shiuchi T, Séi H. Ketone body metabolism and sleep homeostasis in mice. Neuropharmacology. 2014;79:399-404.
3. Chikahisa S, Kodama T, Soya A, Sagawa Y, Ishimaru Y, Séi $\mathrm{H}$, Nishino S. Histamine from brain resident MAST cells promotes wakefulness and modulates behavioral states. PLoS One. 2013;8(10):e78434.

4. Chikahisa S, Tominaga K, Kawai T, Kitaoka K, Oishi K, Ishida N, Rokutan K, Séi H. Bezafibrate, a peroxisome proliferator-activated receptors agonist, decreases body temperature and enhances electroencephalogram delta-oscillation during sleep in mice. Endocrinology. 2008;149(10):5262-71. 